\title{
Cloning and characterization of the gene encoding phosphoketolase in Leuconostoc mesenteroides isolated from kimchi
}

Jung Min Lee ${ }^{1}$, Do-Won Jeong ${ }^{1}$, Ok Kyung Koo ${ }^{1}$, Min Jung Kim ${ }^{1}$, Jong-Hoon Lee ${ }^{2}$, Hae Choon Chang ${ }^{3}$, Jeong Hwan $\mathrm{Kim}^{4}$ \& Hyong Joo Lee ${ }^{1, *}$

${ }^{1}$ School of Agricultural Biotechnology, and Center for Agricultural Biomaterials, Seoul National University, 151-742, Seoul, Republic of Korea

${ }^{2}$ Department of Food Science and Biotechnology, Kyonggi University, 443-760, Suwon, Republic of Korea

${ }^{3}$ Department of Food and Nutrition, Chosun University, 501-759, Kwangju, Republic of Korea

${ }^{4}$ Division of Applied Life Science, Graduate School, Gyeongsang National University, 660-701, Jinju, Republic of Korea

*Author for correspondence (Fax: +82-2-873-5095; E-mail: leehyjo@snu.ac.kr)

Biotechnology Letters (2005) 27: 853-858

DOI $10.1007 / \mathrm{s} 10529-005-6718-2$

The names of the third and fourth author were misrepresented on the title page of the original. 